\title{
Cloaking via anomalous localized resonance. A connection between the localized resonance and the blow up of the power for doubly complementary media
}

\author{
Invisibilité par résonance localisée anormale. Une liaison entre la résonance \\ localisée et l'exposion de la puissance pour les milieux doublement \\ complémentaires
}

\author{
Hoai-Minh Nguyen \\ École polytechnique fédérale de Lausanne, SB MATHAA CAMA, Station 8, CH-1015 Lausanne, Switzerland
}

\section{A R T I C L E I N F O}

\section{Article history:}

Received 25 August 2014

Accepted after revision 21 October 2014

Available online 12 November 2014

Presented by Haïm Brézis

\begin{abstract}
A B S T R A C T
This paper is devoted to the study of cloaking via anomalous localized resonance (CALR) in two and three dimensions in the quasistatic regime. Two key figures of CALR are (i) the localized resonance and (ii) the connection between the localized resonance and the blow up of the power of the fields as the loss goes to 0. An important class of negative index materials for which the localized resonance might appear is the class of (reflecting) complementary media introduced and analyzed in [8-10]. It was shown in [12] that the complementary property of media is not enough to ensure such a connection. In this paper, we introduce a subclass of complementary media called the class of doubly complementary media. This class is rich enough to allow us to do cloaking via anomalous localized resonance for an arbitrary source concentrating on an arbitrary smooth bounded manifold of codimension 1 located in an arbitrary medium. The following three properties are established: 1) CALR appears if and only if the power blows up; 2) the power blows up if the source is "located" near the plasmonic structure; 3 ) the power remains bounded if the source is far away from the plasmonic structure. Property P2), the blow up of the power, is in fact established for reflecting complementary media.

(c) 2014 Académie des sciences. Published by Elsevier Masson SAS. All rights reserved.
\end{abstract}

\section{R É S U M É}

Nous étudions l'invisibilité par résonance localisée anormale (CALR) en deux et trois dimensions en régime quasi-statique. Deux figures principales de CALR sont $i$ ) la résonance localisée et $i i$ ) la liaison entre la résonance localisée et l'exposion de la puissance quand la perte de la matériel tend vers 0 . Une importante classe de matériels de l'indice négatif pour laquel la résonance localisée peut apparaître est la classe de milieux complémentaires introduite et analysée dans [8-10]. Il a été noté dans [12] que la propriété complémentaire ne suffit pas à assurer une telle liaison. Dans cette note, nous introduisons une sous-classe des milieux complémentaires s'appelée la classe des milieux doublement complémentaires. Cette classe est suffisament large pour accomplir l'invisibilité par résonance localisée

E-mail address: hoai-minh.nguyen@epfl.ch. 
anormale une source arbitraire concentrant sur une sous-variété arbitraire de codimension 1 placé dans un milieu arbitraire. Les trois propriétés suivantes sont établies : 1) CALR apparaît si et seulement si la puissance explose; 2) la puissance explose si la source est "placée " près de la structure plasmonique ; 3 ) la puissance reste bornée si la source est loin de la structure plasmonique. Propriété P2), l'explosion de la puissance est en fait établie pour les milieux complémentaires.

(C) 2014 Académie des sciences. Published by Elsevier Masson SAS. All rights reserved.

\section{Introduction}

Negative index materials (NIMs) were first investigated theoretically by Veselago in [17] and were innovated by Nicorovici et al. in [13] and Pendry in [14]. The existence of such materials was confirmed by Shelby et al. in [16]. The study of NIMs has attracted a lot attention in the scientific community thanks to their many possible applications. One of the appealing ones is cloaking via anomalous localized resonance.

Cloaking via anomalous localized resonance (CALR) was discovered by Milton and Nicorovici in [7] and has root from [13] (see also [6]). In [7], Milton and Nicorovici studied the core-shell plasmonic structures in the two-dimensional quasistatic regime in which a circular shell has permittivity $-1+i \delta$, while the core and the matrix, the complement of the core and the shell, have permittivity 1 . Here $\delta$ denotes the loss of the material in the shell. Let $r_{e}$ and $r_{i}$ be the outer and the inner radius of the shell. They showed that there is a critical radius which is $r_{*}:=\left(r_{e}^{3} r_{i}^{-1}\right)^{1 / 2}$ such that a polarizable point dipole is not seen by an observer away from the core-shell structure, hence it is cloaked, if and only if it is within distance $r_{*}$ of the shell; moreover, the power of the field $u_{\delta}$, which is roughly speaking $\delta\left\|u_{\delta}\right\|_{H^{1}}^{2}$, blows up in a bounded neighborhood of the shell. They called this phenomenon "cloaking via anomalous localized resonance". Two key figures of CALR are $i$ ) the localized resonance, i.e., the field blows up in some region and remains bounded in some other, and $i i$ ) the connection between the localized resonance and the blow up of the power of the fields as the loss goes to 0 .

The study of CALR has been mainly based on separation of variables see [1-4,6,7]. In addition to the separation of variables technique, there are two methods suggested by Ammari et al. in [1] and Kohn et al. in [5] to study the blow up of the power. They considered non-radial core-shell structures in which the shell has permittivity $-1+i \delta$ and the core and the matrix have permittivity 1 in the two-dimensional quasistatic regime. In [1], Ammari et al. dealt with arbitrary shells and provided a characterization of sources for which the power blows up. Their characterization is based on the spectrum of a self-adjoint compact operator (Neumann-Poincaré-type operator). In [5], Kohn et al. considered core-shell structures in which the outer shell surface is radially symmetric, but the core is not. Using a variational approach, they showed that the power blows up for a class of sources concentrated on circles within distance $r_{*}=\left(r_{e}^{3} r_{i}^{-1}\right)^{1 / 2}$ of the core-shell structure $B_{r_{e}}$ if the core is inside $B_{r_{i}}$. In [5], they also showed that the power remains bounded for a class of sources concentrated on circles outside $B_{r_{*}}$ if the core is round, inside, and close to $B_{r_{i}}$.

One of the key figures of CALR is the localized resonance. An important class of NIMs for which the localized resonance might appear is the class of reflecting complementary media. This class was introduced and studied in [8]. This work is inspired from the pivotal one of Nicorovici et al. in [13] and from the important suggestion of concept of complementary media due to Ramakrishna et al. in [15]. The localized resonance related to materials in this class in the context of cloaking and superlensing was analyzed in [9,10]. It was shown in [12] that the complementary property of media is not enough to ensure a connection between the blow up of the power and the localized resonance.

In this paper, we introduce a subclass of reflecting complementary media called the class of doubly complementary media. This class is rich enough to allow us to do cloaking via anomalous localized resonance for an arbitrary source concentrating on an arbitrary smooth bounded manifold of codimension 1 placed in an arbitrary medium. The following three properties, which are what one would expect from a structure for which CALR takes place, are established for doubly complementary media in both two and three dimensions:

(P1) CALR appears if and only if the power blows up (Theorem 1);

(P2) the power blows up if the source is "located" near the shell (Theorem 2);

(P3) the power remains bounded if the source is far away from the shell (Proposition 1 and Theorem 3).

In fact, Property P2) is established for reflecting complementary media. These results in this paper are inspired by and imply recent ones of Ammari et al. in [1] and Kohn et al. in [5] and extend theirs to a general class of non-radial core-shell structures in both two and three dimensions.

\section{Statement of the main results}

Let $d=2,3, \Omega$ be a smooth open bounded subset of $\mathbb{R}^{d}$ and let $A$ be a uniformly elliptic matrix-valued function defined in $\Omega$, i.e., 


$$
\frac{1}{\Lambda}|\xi|^{2} \leq\langle A(x) \xi, \xi\rangle \leq \Lambda|\xi|^{2} \quad \forall \xi \in \mathbb{R}^{d}
$$

for a.e. $x \in \Omega$ and for some $0<\Lambda<+\infty$. Let $0<r_{1}<r_{2}<r_{3}$ be such that $B_{r_{3}} \Subset \Omega$. Set

$$
s_{\delta}:= \begin{cases}-1+i \delta & \text { in } B_{r_{2}} \backslash B_{r_{1}} \\ 1 & \text { otherwise. }\end{cases}
$$

Let $f \in L^{2}(\Omega)$ with supp $f \cap B_{r_{2}}=\emptyset$ and let $u_{\delta} \in H_{0}^{1}(\Omega)$ be the unique solution to

$$
\operatorname{div}\left(s_{\delta} A \nabla u_{\delta}\right)=f \quad \text { in } \Omega .
$$

The power (dissipative energy) $E_{\delta}\left(u_{\delta}\right)$ is defined by (see, e.g., [7])

$$
E_{\delta}\left(u_{\delta}\right)=\delta \int_{B_{r_{2}} \backslash B_{r_{1}}}\left|\nabla u_{\delta}\right|^{2} .
$$

From the definition of $u_{\delta}$, one can verify that

$$
\int_{\Omega}\left|\nabla u_{\delta}\right|^{2} \leq C\left(\int_{B_{r_{2}} \backslash B_{r_{1}}}\left|\nabla u_{\delta}\right|^{2}+\|f\|_{L^{2}}\right),
$$

for some positive constants $C$ independent of $f$ and $\delta \in(0,1)$. Let $v_{\delta} \in H_{0}^{1}(\Omega)$ be the unique solution to

$$
\operatorname{div}\left(s_{\delta} A \nabla v_{\delta}\right)=f_{\delta} \quad \text { in } \Omega .
$$

Here

$$
f_{\delta}=c_{\delta} f
$$

and $c_{\delta}$ is the constant such that

$$
\delta \int_{\Omega}\left|\nabla v_{\delta}\right|^{2}=1 .
$$

In this paper, we are interested in a class of $A$, the class of doubly complementary media, for which CALR takes place. In particular, if $E_{\delta}\left(u_{\delta}\right) \rightarrow \infty$ then $v_{\delta} \rightarrow 0$ in the region far away from $B_{r_{2}}$. Before giving the definition of doubly complementary media for a general core-shell structure, let us recall the definition of reflecting complementary media introduced in [8, Definition 1].

Definition 1 (Reflecting complementary media). The media $A$ in $B_{r_{3}} \backslash B_{r_{2}}$ and $-A$ in $B_{r_{2}} \backslash B_{r_{1}}$ are said to be reflecting complementary if there exists a diffeomorphism $F: B_{r_{2}} \backslash \bar{B}_{r_{1}} \rightarrow B_{r_{3}} \backslash \bar{B}_{r_{2}}$ such that

$$
\begin{array}{ll}
F_{*} A=A & \text { for } x \in B_{r_{3}} \backslash \bar{B}_{r_{2}}, \\
F(x)=x & \text { on } \partial B_{r_{2}},
\end{array}
$$

and the following two conditions hold:

1. There exists a diffeomorphism extension of $F$, which is still denoted by $F$, from $B_{r_{2}} \backslash\left\{x_{1}\right\} \rightarrow B_{r_{4}} \backslash \bar{B}_{r_{2}}$ for some $x_{1} \in B_{r_{1}}$ and $r_{3}<r_{4} \leq+\infty{ }^{1}$

2. There exists a diffeomorphism $G: B_{r_{4}} \backslash \bar{B}_{r_{3}} \rightarrow B_{r_{3}} \backslash\left\{x_{2}\right\}$ for some $x_{2} \in B_{r_{3}}$ such that ${ }^{2}$

$$
G(x)=x \quad \text { on } \partial B_{r_{3}},
$$

and

$$
G \circ F: B_{r_{1}} \rightarrow B_{r_{3}} \text { is a diffeomorphism if one sets } G \circ F\left(x_{1}\right)=x_{2} .
$$

As noted in [8], conditions (5) and (6) are the main assumptions in the definition of complementary media. The term "reflecting" in the definition comes from (6) and the fact that $B_{r_{1}} \subset B_{r_{2}} \subset B_{r_{3}}$. Conditions 1 and 2 in the definition are mild assumptions. Introducing $G$ in the definition makes the analysis more accessible, see [8-10].

We are ready to introduce the concept of doubly complementary media.

\footnotetext{
$1 \quad B_{r_{4}}$ denotes $\mathbb{R}^{d}$ when $r_{4}=+\infty$.

2 In (6) and (7), $F$ and $G$ denote some diffeomorphism extensions of $F$ and $G$ in a neighborhood of $\partial \Omega_{2}$ and of $\partial \Omega_{3}$.
} 
Definition 2 (Doubly complementary media). The medium $s_{0} A$ is said to be doubly complementary if and only if, for some $r_{3}>r_{2}$ with $B_{r_{3}} \Subset \Omega, A$ in $B_{r_{3}} \backslash B_{r_{2}}$ and $-A$ in $B_{r_{2}} \backslash B_{r_{1}}$ are reflecting complementary, and

$$
F_{*} A=G_{*} F_{*} A=A \text { in } B_{r_{3}} \backslash B_{r_{2}},
$$

for some $F$ and $G$ from Definition 1 .

Define $\Omega_{0}=(G \circ F)^{-1}\left(B_{r_{3}} \backslash B_{r_{2}}\right)$. Then $A$ in $\Omega_{0}$ and $-A$ in $B_{r_{2}} \backslash B_{r_{1}}$ are reflecting complementary in the sense that

$$
\left(F^{-1} \circ G \circ F\right)_{*} A=\left(F^{-1}\right)_{*} G_{*} F_{*} A=\left(F^{-1}\right)_{*} F_{*} A=A \quad \text { in } B_{r_{2}} \backslash B_{r_{1}}
$$

and

$$
F^{-1} \circ G \circ F(x)=x \quad \text { on } \partial B_{r_{1}}
$$

since $G(x)=x$ on $\partial B_{r_{3}}$ and $F\left(\partial B_{r_{1}}\right)=\partial B_{r_{3}}$. Hence $-A$ in $B_{r_{2}} \backslash B_{r_{1}}$ is complementary to $A$ in $B_{r_{3}} \backslash B_{r_{2}}$ and $A$ in $\Omega_{0}$. This is the reason for which media satisfying (9) are called doubly complementary media.

Remark 1. We assumed that $B_{r_{2}} \backslash B_{r_{1}}$ is the region of negative index and the region $B_{r_{3}} \backslash B_{r_{2}}$ is its reflecting complementary medium. Since $A$ is only assumed symmetric and uniformly elliptic in these regions, the radial assumption of these regions is mainly for the simplicity of presentation.

The first main result of the paper, which implies Property P1), is:

Theorem 1. Let $d=2,3, g_{n} \in L^{2}(\Omega)$ with supp $g_{n} \subset \Omega \backslash B_{r_{2}},\left(\delta_{n}\right)$ be a positive sequence converging to 0 . Assume that A satisfies (1) and (9), and $A \in C^{2}\left(\overline{B_{r_{3}} \backslash B_{r_{2}}}\right)$ and let $v_{n} \in H_{0}^{1}(\Omega)$ be the unique solution to

$$
\operatorname{div}\left(s_{\delta_{n}} A \nabla v_{n}\right)=g_{n} \text { in } \Omega
$$

Assume that $g_{n} \rightarrow g$ weakly in $L^{2}(\Omega)$ for some $g \in L^{2}(\Omega)$, and $\lim _{n \rightarrow \infty} \delta_{n}\left\|\nabla v_{n}\right\|_{L^{2}(\Omega)}^{2}=0$. Then $v_{n} \rightarrow v$ weakly in $H^{1}\left(\Omega \backslash B_{r_{3}}\right)$ where $v \in H_{0}^{1}(\Omega)$ is the unique solution to

$$
\operatorname{div}(\hat{A} \nabla v)=g \quad \text { in } \Omega \text {. }
$$

Here

$$
\hat{A}= \begin{cases}A & \text { in } \Omega \backslash B_{r_{3}}, \\ G_{*} F_{*} A & \text { in } B_{r_{3}} .\end{cases}
$$

Let us explain how Theorem 1 implies the equivalence between the blow up of the power and the CALR. Suppose that the power of $u_{\delta_{n}}$ blows up ${ }^{3}$, i.e.,

$$
\lim _{n \rightarrow \infty} \delta_{n}\left\|\nabla u_{\delta_{n}}\right\|_{L^{2}\left(B_{r_{2}} \backslash B_{r_{1}}\right)}^{2}=+\infty .
$$

It follows from the definition of $v_{\delta_{n}}$ in (4) that $v_{\delta_{n}} \in H_{0}^{1}(\Omega)$ is the unique solution to

$$
\operatorname{div}\left(s_{\delta_{n}} A \nabla v_{\delta_{n}}\right)=\alpha_{n} f \quad \text { in } \Omega
$$

moreover,

$$
\delta_{n} \int_{\Omega}\left|\nabla v_{\delta_{n}}\right|^{2}=1 .
$$

Since $\alpha_{n} f \rightarrow 0$ by (11), one derives from Theorem 1 that $v_{\delta_{n}} \rightarrow 0$ in $\Omega \backslash B_{r_{3}}$ : the source $\alpha_{n} f$ is not seen by observers far away from the shell: the source is cloaked (see also [1] for a detailed explanation of this property). We note that localized resonance takes place since (12) holds and $v_{\delta_{n}} \rightarrow 0$ in $\Omega \backslash B_{r_{3}}$. If the power of $u_{\delta_{n}}$ remains bounded, then $u_{\delta_{n}} \rightarrow u$ weakly in $H^{1}\left(\Omega \backslash B_{r_{3}}\right)$ where $u \in H_{0}^{1}(\Omega)$ is the unique solution to $\operatorname{div}(\hat{A} \nabla u)=f$ in $\Omega$. The source is not cloaked. Property P1) is proved.

The second main result of the paper, which implies Property P2), is

\footnotetext{
${ }^{3}$ Recall that $u_{\delta}$ is defined by (3).
} 
Theorem 2. Let $d=2,3,0<R_{1}<R_{2}<+\infty$, M be a uniformly elliptic matrix-valued function defined in $B_{R_{2}} \backslash B_{R_{1}}$, and let $f$, $g \in$ $L^{2}\left(B_{R_{2}} \backslash B_{R_{1}}\right)$. Assume that $M$ is Lipschitz and let $U_{\delta}, V_{\delta} \in H^{1}\left(B_{R_{2}} \backslash B_{R_{1}}\right)$ be such that

$$
\operatorname{div}\left(M \nabla U_{\delta}\right)=g \quad \text { in } B_{R_{2}} \backslash B_{R_{1}}, \quad \operatorname{div}\left(M \nabla V_{\delta}\right)=h \quad \text { in } B_{R_{2}} \backslash B_{R_{1}},
$$

and

$$
U_{\delta}=V_{\delta} \quad \text { on } \partial B_{R_{1}}, \quad M \nabla U_{\delta} \cdot \eta=(1-i \delta) M \nabla V_{\delta} \cdot \eta \quad \text { on } \partial B_{R_{1}} .
$$

Then there exists a constant $R_{*} \in\left(R_{1}, R_{2}\right)$ depending on $R_{1}, R_{2}$, the Lipschitz constant and the elliptic constant of $M$, but independent of $\delta, f$, and $g$ such that if there is no $v \in H^{1}\left(B_{R_{*}} \backslash B_{R_{1}}\right)$ with the properties

$$
\operatorname{div}(M \nabla W)=g-h \quad \text { in } B_{R_{*}} \backslash B_{R_{1}}, \quad W=0 \quad \text { on } \partial B_{R_{1}}, \quad \text { and } \quad M \nabla W \cdot \eta=0 \quad \text { on } \partial B_{R_{1}},
$$

then

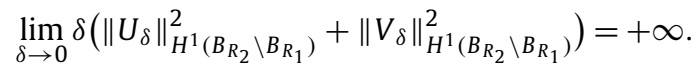

Assume in addition that $M=I$ in $B_{R_{2}} \backslash B_{R_{1}}$, then

$R_{*}$ can be taken by any number less than $\sqrt{R_{1} R_{2}}$.

Property P2), which is also valid for reflecting complementary media (in particular (5) and (6) hold), can be derived from Theorem 2 as follows. Define $v_{\delta}=u_{\delta} \circ F$ in $B_{r_{3}} \backslash B_{r_{2}}$. By [8, Lemma 2] (a change of variables formula), one has:

$$
v_{\delta}=u_{\delta} \quad \text { and } \quad A \nabla u_{\delta} \cdot \eta=(1-i \delta) A \nabla v_{\delta} \cdot \eta \quad \text { on } \partial B_{r_{2}} .
$$

Applying Theorem 2 for $R_{1}=r_{2}, R_{2}=r_{3}, U_{\delta}=u_{\delta}$, and $V_{\delta}=v_{\delta}$, one reaches Property P2) for reflecting complementary media.

Remark 2. From Theorem 2, by taking $A=I$, one rediscovers, by a different approach, the result of Ammari et al. in [1] and also of Kohn et al. in [5] on the blow up of the power as mentioned in the Introduction.

The following result, which motivated our definition of doubly complementary media and implies Property P3), was established in [8] (see [8, Theorems 1 and 2 and Corollary 1]):

Proposition 1. Let $d=2,3, f \in L^{2}(\Omega)$ with supp $f \cap B_{r_{3}}=\emptyset$. Assume that A satisfies (1) and $(9)$, and $A \in C^{1}\left(\overline{B_{r_{3}} \backslash B_{r_{2}}}\right)$. Then $\left\|u_{\delta}\right\|_{H^{1}(\Omega)}$ remains bounded as $\delta \rightarrow 0$.

The third main result of this paper is the following qualitative estimate for Property P3).

Theorem 3. Let $d=2,3, f \in L^{2}(\Omega)$ with supp $f \subset \Omega \backslash B_{r_{2}}$. Assume that $A$ satisfies (1) and (9), and $A=I$ in $B_{r_{3}} \backslash B_{r_{2}}$. Let $u_{\delta} \in H_{0}^{1}(\Omega)$ be the unique solution to

$$
\operatorname{div}\left(s_{\delta} A \nabla u_{\delta}\right)=f \quad \text { in } \Omega
$$

Assume that there exists $v \in H^{1}\left(B_{r_{0}} \backslash B_{r_{2}}\right)$ for some $r_{0}>\sqrt{r_{2} r_{3}}$ with the properties

$$
\operatorname{div}(A \nabla v)=f \quad \text { in } B_{r_{0}} \backslash B_{r_{2}}, \quad v=0 \quad \text { on } \partial B_{r_{2}}, \quad \text { and } \quad A \nabla v \cdot \eta=0 \quad \text { on } \partial B_{r_{2}} .
$$

Then

$$
\limsup _{\delta \rightarrow 0} \delta\left\|u_{\delta}\right\|_{H^{1}(\Omega)}^{2}<+\infty
$$

Remark 3. From Theorem 3, one rediscovers, by a different approach, the results of Ammari et al. [1] and also of Kohn et al. [5] on the boundedness of the power by taking $A=I$ in $\Omega .{ }^{4}$

\footnotetext{
4 In a recent discussion with Graeme Milton, we realize that the result of Kohn et al. on the boundedness of the power [5, Theorem 5.3] can be derived from the radial setting using the Mobius transformation. We are grateful to him for the discussion.
} 
The proofs of the results presented in this paper are based on several new techniques and observations. One of the difficulties in the study of CALR is the localized resonance. To handle this difficulty, we extend the reflecting and the removing of localized singularity techniques introduced in [8-10], and implement the separation of variables for Cauchy problems for a general structure, which is one of the keys of our analysis. The condition $A \in C^{2}\left(\overline{B_{r_{3}} \backslash B_{r_{1}}}\right)$ is required from the technique of separation of variables. The proof of Theorem 2, which is one of new observations, is based on three sphere inequalities. The theory presented here allows us to do cloaking via anomalous localized resonance for an arbitrary source concentrating on an arbitrary smooth bounded manifold of codimension 1 placed in an arbitrary medium will be given in [11]. The two key points behind this fact are as follows. Firstly given arbitrary $A$ in $B_{r_{3}} \backslash B_{r_{2}}$, one can choose $A$ in $B_{r_{2}}$ such that $s_{0} A$ is a doubly complementary medium. For example, let $F$ and $G$ be the Kelvin transform with respect to $\partial B_{r_{2}}$ and $\partial B_{r_{3}}$ respectively. Define $A$ by $F_{*}^{-1} A$ in $B_{r_{2}} \backslash B_{r_{1}}$ with $r_{1}=r_{2}^{2} / r_{3}$ and $F_{*}^{-1} G_{*}^{-1} A$ in $F^{-1} \circ G^{-1}\left(B_{r_{3}} \backslash B_{r_{2}}\right)=B_{r_{2}} \backslash B_{r_{2}^{3} / r_{3}^{2}}$. Then $s_{0} A$ is a doubly complementary medium. Secondly, there is no $v$ as in Theorem 2 for such a concentrating source as the source is located near the shell.

The details of the proofs of the results presented here are given in [11].

\section{Acknowledgements}

The research was partially supported by NSF grant DMS-1201370 and by the Alfred P. Sloan Foundation.

\section{References}

[1] H. Ammari, G. Ciraolo, H. Kang, H. Lee, G.W. Milton, Spectral theory of a Neumann-Poincaré-type operator and analysis of cloaking due to anomalous localized resonance, Arch. Ration. Mech. Anal. 218 (2013) 667-692.

[2] H. Ammari, G. Ciraolo, H. Kang, H. Lee, G.W. Milton, Anomalous localized resonance using a folded geometry in three dimensions, Proc. R. Soc. Lond. Ser. A 469 (2013) 20130048.

[3] H. Ammari, G. Ciraolo, H. Kang, H. Lee, G.W. Milton, Spectral theory of a Neumann-Poincaré-type operator and analysis of cloaking due to anomalous localized resonance II, Contemp. Math. 615 (2014) 1-14.

[4] G. Bouchitté, B. Schweizer, Cloaking of small objects by anomalous localized resonance, Q. J. Mech. Appl. Math. 63 (2010) $437-463$.

[5] R.V. Kohn, J. Lu, B. Schweizer, M.I. Weinstein, A variational perspective on cloaking by anomalous localized resonance, Commun. Math. Phys. 328 (2014) $1-27$.

[6] G.M. Milton, N.P. Nicorovici, R.C. McPhedran, V.A. Podolskiy, A proof of superlensing in the quasistatic regime, and limitations of superlenses in this regime due to anomalous localized resonance, Proc. R. Soc. Lond. Ser. A 461 (2005) 3999-4034.

[7] G.W. Milton, N.P. Nicorovici, On the cloaking effects associated with anomalous localized resonance, Proc. R. Soc. Lond. Ser. A 462 (2006) $3027-3059$.

[8] H.M. Nguyen, Asymptotic behavior of solutions to the Helmholtz equations with sign changing coefficients, Trans. Amer. Math. Soc. (2014), in press, http://arxiv.org/abs/1204.1518.

[9] H.M. Nguyen, Cloaking using complementary media in the quasistatic regime, http://arxiv.org/pdf/1310.5483.pdf, 2013.

[10] H.M. Nguyen, Superlensing using complementary media, Ann. Inst. Henri Poincaré, Anal. Non Linéaire (2014), http://dx.doi.org/10.1016/j.anihpc. 2014.01.004, in press.

[11] H.M. Nguyen, Cloaking via anomalous localized resonance for doubly complementary media in the quasistatic regime, http://arxiv.org/abs/1407.7977, 2014.

[12] H.M. Nguyen, H.L. Nguyen, Complete resonance and localized resonance in plasmonic structures, ESAIM Math. Model. Numer. Anal. (2014), to appear, http://arxiv.org/abs/1310.3633.

[13] N.A. Nicorovici, R.C. McPhedran, G.M. Milton, Optical and dielectric properties of partially resonant composites, Phys. Rev. B 49 (1994) $8479-8482$.

[14] J.B. Pendry, Negative refraction makes a perfect lens, Phys. Rev. Lett. 85 (2000) 3966-3969.

[15] S.A. Ramakrishna, J.B. Pendry, Focusing light using negative refraction, J. Phys. Condens. Matter 15 (2003) 6345.

[16] R.A. Shelby, D.R. Smith, S. Schultz, Experimental verification of a negative index of refraction, Science 292 (2001) 77-79.

[17] V.G. Veselago, The electrodynamics of substances with simultaneously negative values of $\varepsilon$ and $\mu$, Usp. Fiz. Nauk 92 (1964) 517-526. 